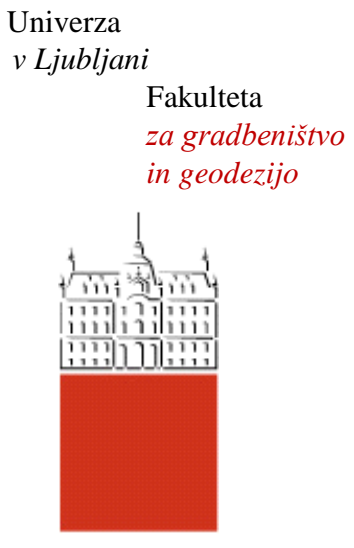

Jamova cesta 2

1000 Ljubljana, Slovenija

http://www3.fgg.uni-lj.si/

\section{DRUGG - Digitalni repozitorij UL FGG http://drugg.fgg.uni-lj.si/}

Ta članek je avtorjeva zadnja recenzirana različica, kot je bila sprejeta po opravljeni recenziji.

Prosimo, da se pri navajanju sklicujte na bibliografske podatke, kot je navedeno:
University
of Ljubljana

Faculty of Civil and Geodetic Engineering

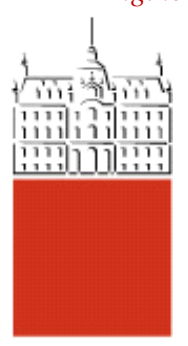

Jamova cesta 2 SI - 1000 Ljubljana, Slovenia http://www3.fgg.uni-lj.si/en/

DRUGG - The Digital Repository http://drugg.fgg.uni-lj.si/

This version of the article is author's manuscript as accepted for publishing after the review process.

When citing, please refer to the publisher's bibliographic information as follows:

Zupan, E., Saje, M., Zupan, D. 2009. The wavelet-based theory of spatial naturally curved and twisted linear beams. Computational mechanics 43,5: 675-686. DOI: 10.1007/s00466008-0337-4. 


\title{
The wavelet-based theory of spatial naturally curved and twisted linear beams
}

\author{
Eva Zupan · Dejan Zupan · Miran Saje
}

Received: date / Accepted: date

\begin{abstract}
The paper presents the wavelet-based discretization of the linearized finite-strain beam theory which assumes small displacements, rotations and strains but is capable of considering an arbitrary initial geometry and material behaviour. In the numerical solution algorithm, we base our derivations on the vector of strain measures as the only unknown functions in a finite element. In such a way the determination of the beam quantities does not require the differentiation. This is an important advantage which allows a wider range of shape functions. In the present paper, the classical polynomial interpolation is compared to scaling and wavelet function interpolations. The computational efficiency of the method is demonstrated by analyzing initially curved and twisted beams.
\end{abstract}

Keywords wavelets - scaling functions - shape functions · linear beam theory $\cdot$ discretization

\section{Introduction}

The wavelets have received an increased attention in the last decade in various engineering disciplines. They have proven to provide a suitable mathematical background for signal processing, processing of images, pattern recognition, diagnosing and monitoring the disturbances, and similar problems. The generality of their applicability stands directly on the attractive properties the sets of wavelets have: periodicity, orthogonality and linear independency. For the theoretical foundation of the wavelet theory, the reader is referred to the mammographies by Chui [2] and Daubechies [3].

The finite element method was successfully combined with the wavelet theory in numerical solutions to Dirichlet problem [18] and Navier-Stokes equations [17]. Several re-

Address(es) of author(s) should be given cent publications deal with the wavelet implementation in the finite element structural analysis, e.g. [4], [7], [10], [21].

We would like to point out that there exists a large number of types of scaling and wavelet functions, which, unfortunately, could not be expressed explicitly in a general way and do not have proper interpolation properties. That is why the implementation of the wavelets in the finite-element theories is non-trivial and often demands some additional theoretical work to be done. One of the implementations is the wavelet-Galerkin method, which demands the shape functions to be expressed in a form of a product of wavelet functions and wavelet coefficients. In such an approach, the relation between the wavelet coefficients and the physical quantities is non-trivial, which makes it difficult to treat element boundary conditions. Alternatively, an additive-type, splinewavelet-based interpolation was proposed by Han, Ren and Huang [4] to resolve this difficulty for conventional displacement-based finite elements.

In the present paper we follow the idea by Han, Ren and Huang and incorporate the additive-type wavelets in the strain based finite-element formulation of spatial beams. In contrast to [4] the present formulation is based on the variant of wavelets presented by Prestin and Quak [11], [12]. The related set of the scaling functions (of any order) and the corresponding set of wavelets not only preserve the properties of the wavelets (they represent the orthonormal base functions), but also possesses two additional properties of an utmost importance in the finite-element implementation: they can be expressed explicitly and have interpolation properties.

There are several reasons for incorporating non-standard shape functions into beam theories. It is well known that in standard interpolation of unknown functions with Lagrange polynomials higher order approximation provides better results, especially in the case of curved beams, see e.g. [5]. It is however not often discussed the use of high order Lagrange 
polynomial might lead to the convergence problems. In contrast to polynomials the wavelet functions are expected to be numerically stable regardless the order of approximation, to better describe local behaviour and are suitable for mesh refinement. The use of wavelets could be of even more beneficial in dynamic problems.

In the present paper the wavelet-based discretization will be applied to the linearized finite-strain beam theory [23] which assumes small displacements, rotations and strains but is capable of considering an arbitrary initial geometry and material behaviour. In the numerical solution algorithm, we base our derivations on the vector of strain measures as the only unknown functions in a finite element. In such a way the determination of the beam quantities does not require any differentiation. This is an important advantage compared to formulations where the derivatives of the shape functions are needed. The efficiency and numerical stability of our approach will be proved by numerical examples.

\section{Scaling and wavelet interpolation}

\subsection{Scaling functions}

We follow the approach of Quak [12], and Prestin and Quak [11], and introduce the scaling functions

$\phi_{j, n}(x)=\phi_{j, 0}\left(x-\frac{n \pi}{2^{j}}\right), \quad$ for $n=0, \ldots, 2^{j+1}-1$,

where

$\phi_{j, 0}(x)=\frac{1}{2^{2 j+2}} \frac{\sin ^{2}\left(2^{j} x\right)}{\sin ^{2}(x / 2)}$,

for $x \neq 2 k \pi$ and $\phi_{j, 0}(x)=1$ if $x=2 k \pi ; k$ is an arbitrary integer and $n$ a non-negative integer.

We will denote by $V_{j}$ the vector spaces, spanned by scaling functions $\phi_{j, n}$ :

$V_{j}=\operatorname{span}\left\{\phi_{j, n} ; n=0, \ldots, 2^{j+1}-1\right\}$.

$V_{j}$ are the vector subspaces of the $2 \pi$-periodic square-integrable functions $L_{2 \pi}^{2}$. It is easy to see that dimension of each space $V_{j}$ equals $2^{j+1}$ and that the spaces $V_{j}$ are nested $\left(V_{j} \subset V_{j+1}\right)$. The interpolatory properties follow directly from the definition of scaling functions. If we define a set of nodes on the interval $[0,2 \pi)$

$x_{j, m}=\left\{\frac{m \pi}{2^{j}} ; m=0, \ldots, 2^{j+1}-1\right\}$,

where $m$ is non-negative integer, we can observe that

$\phi_{j, n}\left(x_{j, m}\right)=\left\{\begin{array}{l}1, \text { if } n=m \\ 0, \text { otherwise }\end{array}=\delta_{n, m}\right.$.

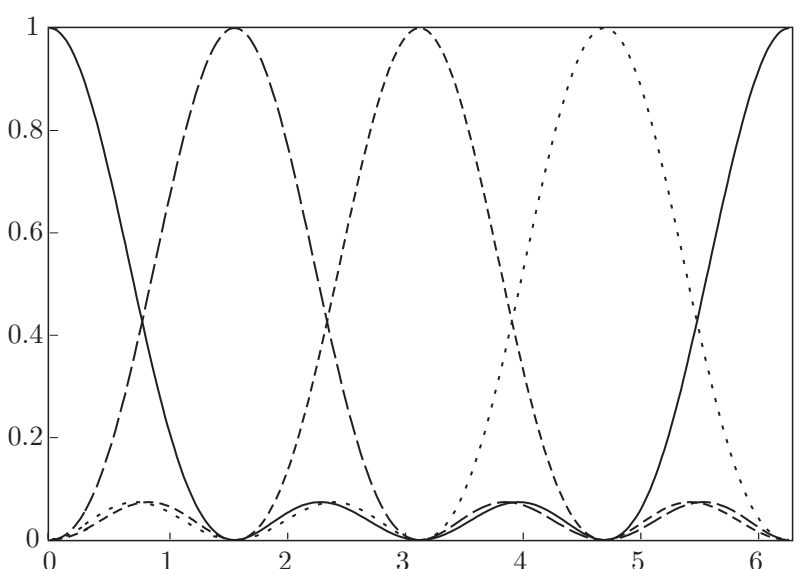

Fig. 1 Scaling functions for $j=1$.

Another interesting and useful property of the scaling functions concerns the change of index in discrete points, e.g.

$$
\begin{aligned}
\phi_{j, n} & \left(\frac{2 m+1}{2^{j+1}} \pi\right)=\phi_{j, 0}\left(\frac{2 m+1}{2^{j+1}} \pi-\frac{n \pi}{2^{j}}\right) \\
& =\phi_{j, 0}\left(\frac{2 m-2 n+1}{2^{j+1}} \pi\right)=\phi_{j, 0}\left(\frac{2 n-2 m-1}{2^{j+1}} \pi\right) \\
& =\phi_{j, 0}\left(\frac{2 n-1}{2^{j+1}} \pi-\frac{m \pi}{2^{j}}\right) .
\end{aligned}
$$

Hence

$\phi_{j, n}\left(\frac{2 m+1}{2^{j+1}} \pi\right)=\phi_{j, m}\left(\frac{2 n-1}{2^{j+1}} \pi\right)$.

Due to the interpolatory properties of $\phi_{j, n}$, we can define the interpolation of an arbitrary real-valued $L_{2 \pi}^{2}$ function $f$

$L_{j}^{\mathrm{s}} f(x)=\sum_{n=0}^{2^{j+1}-1} f\left(x_{j, n}\right) \phi_{j, n}(x)$.

For illustration, the graphs of four base functions spanning $V_{1}$ are presented in Figure 1. We would like to emphasize that the sum of the base functions equals 1 at each level $j$. This means that a constant can be exactly approximated by the above type of interpolation.

\subsection{Wavelet functions}

In accordance with the wavelet theory, the wavelets are constructed as the linear combination of scaling functions:

$\psi_{j, n}(x)=2 \phi_{j+1,2 n+1}(x)-\phi_{j, n}\left(x-\frac{\pi}{2^{j+1}}\right)$.

It is obvious from (8) that $\psi_{j, n} \in V_{j+1}$ and we can observe that $\psi_{j, n}$ also have interpolatory properties. The corresponding nodes are the midpoints between $x_{j, m}$; they represent $2^{j+1}$ equally-spaced points on the interval $[0,2 \pi)$ :

$y_{j, m}=\left\{\frac{(2 m+1) \pi}{2^{j+1}} ; m=0, \ldots, 2^{j+1}-1\right\}$. 
At the set of points $y_{j, m}$ we have

$\phi_{j, n}\left(y_{j, m}\right)=\left\{\begin{array}{l}1, \text { if } n=m \\ 0, \text { otherwise. }\end{array}\right.$

Consequently, the interpolatory properties of $\phi_{j+1,2 n+1}$ result in a simple expression for the values of wavelets at $x_{j, m}$ :

$\psi_{j, n}\left(x_{j, m}\right)=-\phi_{j, n}\left(\frac{m \pi}{2^{j}}-\frac{\pi}{2^{j+1}}\right)=-\phi_{j, n}\left(\frac{(2 m-1) \pi}{2^{j}}\right)$.

In addition to the vector spaces, spanned by the scaling functions, we define the spaces $W_{j}$, spanned by wavelets $\psi_{j, n}$ :

$W_{j}=\operatorname{span}\left\{\psi_{j, n} ; n=0, \ldots, 2^{j+1}-1\right\}$.

Due to the interpolation properties of wavelets, we can also define the interpolation of an arbitrary real-valued $L_{2 \pi}^{2}$ function $f$ as

$L_{j}^{\mathrm{w}} f(x)=\sum_{n=0}^{2^{j+1}-1} f\left(x_{j, n}\right) \psi_{j, n}(x)$.

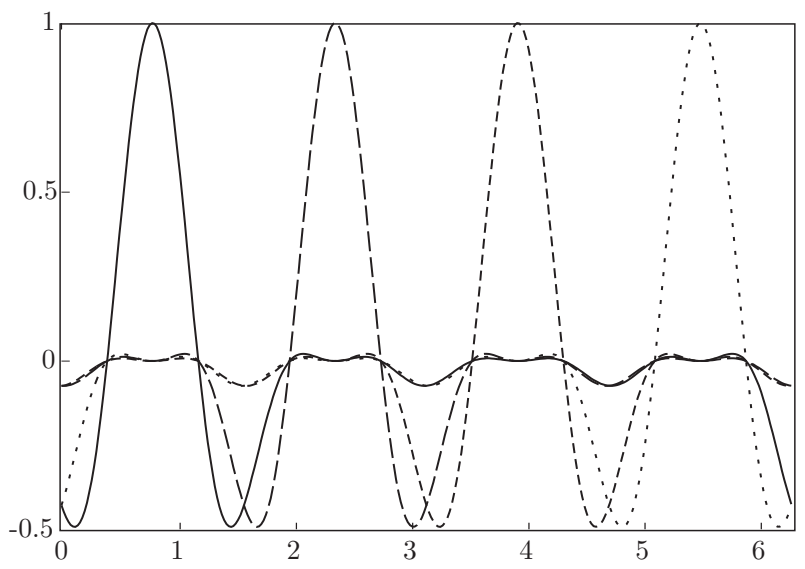

Fig. 2 Wavelet functions for $j=1$.

The wavelets for $j=1$ are shown in Figure 2. It is interesting to observe that the sum of the wavelet functions equals a cosine function, i.e. $\sum_{n=0}^{2^{j+1}-1} \psi_{j, n}(x)=-\cos \left(2^{j+2} x\right)$. This result can be of an utmost importance in the analysis of naturally curved and twisted rods, where the cosine function is typically a part of the analytical solution (see [23] for further details).

\subsection{Decomposition sequences}

The main motivation in decomposition sequences is to use both scaling functions and wavelets, as the shape functions. Thus we are capable of accurately solving the problem, whose solution can be expressed as the linear combination of a constant and a cosine function. We now explain the necessary mathematical background.

From the definition (8) follows that the vector space, spanned by wavelets, is the subspace of one degree higher vector space, spanned by scaling functions, i.e. $W_{j} \subset V_{j+1}$. Moreover, $W_{j}$ is the orthogonal complement of $V_{j}$, which is usually written in the form [3]

$V_{j+1}=V_{j} \oplus W_{j}$,

where $\oplus$ denotes the orthogonal sum of vector spaces. The orthogonality of $V_{j}$ and $W_{j}$ means that the scalar products of the base functions in $L_{2 \pi}^{2}$ are zero:

$\left\langle\psi_{j, n}, \phi_{j, m}\right\rangle=\frac{1}{2 \pi} \int_{0}^{2 \pi} \psi_{j, n}(x) \phi_{j, m}(x) d x=0$.

We have found out that both $\left\{\phi_{j+1, n} ; n=0, \ldots, 2^{j+2}-1\right\}$ and $\left\{\phi_{j, n}, \psi_{j, n} ; n=0, \ldots, 2^{j+1}-1\right\}$ are orthonormal bases of the vector space $V_{j+1}$. Thus an arbitrary vector from $V_{j+1}$ can be expressed in either of the two bases. For the interpolation of an arbitrary function with combined scaling and wavelet functions, it suffices to find the connection between the two bases.

Since $V_{j} \subset V_{j+1}$ there exist such coefficients $p_{m}^{j, n}$ that $\phi_{j, n}=\sum_{m} p_{m}^{j, n} \phi_{j+1, m}$. From the properties of the interpolatory operator (7) we have

$\phi_{j, n}(x)=L_{j+1}^{\mathrm{s}} \phi_{j, n}(x)=\sum_{m=0}^{2^{j+2}-1} \phi_{j, n}\left(\frac{m \pi}{2^{j+1}}\right) \phi_{j+1, m}(x)$.

Since $\phi_{j, n}\left(\frac{2 s \pi}{2^{j+1}}\right)=\delta_{n, s}$, it follows

$\phi_{j, n}(x)=\phi_{j+1,2 n}+\sum_{s=0}^{2^{j+1}-1} \phi_{j, n}\left(\frac{2 s+1}{2^{j+1}} \pi\right) \phi_{j+1,2 s+1}$.

Similarly, as $W_{j} \subset V_{j+1}$ we can find coefficients $q_{m}^{j, n}$ such that $\psi_{j, n}=\sum_{m} q_{m}^{j, n} \phi_{j+1, m}$ :

$$
\begin{aligned}
\psi_{j, n}(x)=2 \phi_{j+1,2 n+1}(x)-\phi_{j, n}\left(x-\frac{\pi}{2^{j+1}}\right) \\
=2 \phi_{j+1,2 n+1}(x)-\sum_{m=0}^{2^{j+2}-1} \phi_{j, n}\left(\frac{m \pi}{2^{j+1}}\right) \phi_{j+1, m}\left(x-\frac{\pi}{2^{j+1}}\right) \\
=2 \phi_{j+1,2 n+1}(x)-\sum_{m=0}^{2^{j+2}-1} \phi_{j, n}\left(\frac{m \pi}{2^{j+1}}\right) \phi_{j+1, m+1}(x) \\
=\phi_{j+1,2 n+1}(x)-\sum_{s=0}^{2^{j+1}-1} \phi_{j, n}\left(\frac{2 s+1}{2^{j+1}} \pi\right) \phi_{j+1,2 s+2}(x) .
\end{aligned}
$$

Again, the property $\phi_{j, n}\left(\frac{2 s \pi}{2^{j+1}}\right)=\delta_{n, s}$ was used. The periodicity of scaling functions yields

$\phi_{j, n}\left(\frac{2^{j+2}-1}{2^{j+1}} \pi\right) \phi_{j+1,2^{j+2}}(x)=\phi_{j, n}\left(-\frac{\pi}{2^{j+1}}\right) \phi_{j+1,0}(x)$, 
which finally results in

$\psi_{j, n}(x)=\phi_{j+1,2 n+1}(x)-\sum_{s=0}^{2^{j+1}-1} \phi_{j, n}\left(\frac{2 s-1}{2^{j+1}} \pi\right) \phi_{j+1,2 s}(x)$.

Further, if we take into account the index transformation (6), the result reads

$\psi_{j, n}(x)=\phi_{j+1,2 n+1}(x)-\sum_{s=0}^{2^{j+1}-1} \phi_{j, s}\left(\frac{2 n+1}{2^{j+1}} \pi\right) \phi_{j+1,2 s}(x)$.

Let $\boldsymbol{\phi}_{j}$ denote the vector $\left[\phi_{j, 0}, \phi_{j, 1}, \ldots, \phi_{j, 2^{j+1}-1}\right]^{T}$ and $\boldsymbol{\psi}_{j}$ the vector $\left[\psi_{j, 0}, \psi_{j, 1}, \ldots, \psi_{j, 2^{j+1}-1}\right]^{T}$. Let permutation $P$ reorder the components in the vector of scaling functions $\boldsymbol{\phi}_{j+1}$ with respect to even and odd indices $n$ :

$$
\begin{aligned}
P \boldsymbol{\phi}_{j+1}= & {\left[\phi_{j+1,0}, \ldots, \phi_{j+1,2 s}, \ldots, \phi_{j+1,2^{j+2}-2},\right.} \\
& \left.\phi_{j+1,1}, \ldots, \phi_{j+1,2 s+1}, \ldots, \phi_{j+1,2^{j+2}-1},\right]^{T} .
\end{aligned}
$$

Then we are able to re-write the results (16) and (17) in a more convenient matrix form:

$$
\left[\begin{array}{l}
\boldsymbol{\phi}_{j} \\
\boldsymbol{\psi}_{j}
\end{array}\right]=\mathbf{D}_{j} P \boldsymbol{\phi}_{j+1} \text {. }
$$

$\mathbf{D}_{j}$ is the so-called reconstruction matrix, which has for the chosen wavelets the following form

$\mathbf{D}_{j}=\left[\begin{array}{cc}\mathbf{I}_{j} & \mathbf{Z}_{j} \\ -\mathbf{Z}_{j}^{T} & \mathbf{l}_{j}\end{array}\right]$,

where $\mathbf{I}_{j}$ is the identity matrix of size $2^{j+1}-1$ and the elements of $\mathbf{Z}_{j}$ are given by

$\mathbf{Z}_{j}(n, s)=\phi_{j, n}\left(\frac{2 s+1}{2^{j+1}} \pi\right)$.

The argument $(n, s)$ denotes the element of matrix in row $n$ and column $s$.

An arbitrary function $f_{j+1} \subset V_{j+1}$ can be uniquely written as

$f_{j+1}=f_{j}+g_{j}, \quad f_{j} \subset V_{j}, g_{j} \subset W_{j}$.

If we express the functions with respect to the bases of the corresponding vector spaces, we have

$$
\begin{aligned}
f_{j+1} & =\sum_{n=0}^{2^{j+2}-1} a_{n}^{j+1} \boldsymbol{\phi}_{j+1, n}(x)=\mathbf{a}_{j+1}^{T} \boldsymbol{\phi}_{j+1}, \\
f_{j} & =\sum_{n=0}^{2^{j+1}-1} a_{n}^{j} \phi_{j, n}(x)=\mathbf{a}_{j}^{T} \boldsymbol{\phi}_{j}, \\
g_{j} & =\sum_{n=0}^{2^{j+1}-1} b_{n}^{j} \psi_{j, n}(x)=\mathbf{b}_{j}^{T} \boldsymbol{\psi}_{j} .
\end{aligned}
$$

By comparing the coefficients and taking (19) into account, it yields

$$
P \mathbf{a}_{j+1}=\mathbf{D}_{j}^{T}\left[\begin{array}{l}
\mathbf{a}_{j} \\
\mathbf{b}_{j}
\end{array}\right] \text {. }
$$

The above result represents one step of the decomposition. If repeated, we obtain the wavelet coefficients $\mathbf{b}_{\eta}$ for $\eta=$ $0, \ldots, j$ and a pair of scaling coefficients $\mathbf{a}_{0}$. In such a way the interpolation is expressed with wavelets of various order and the lowest level scaling functions. Shape functions for the decomposition-based interpolation of order $2^{3}$ are presented in Figure 3. It is obvious that the localizations can particularly be well described by such an interpolation.

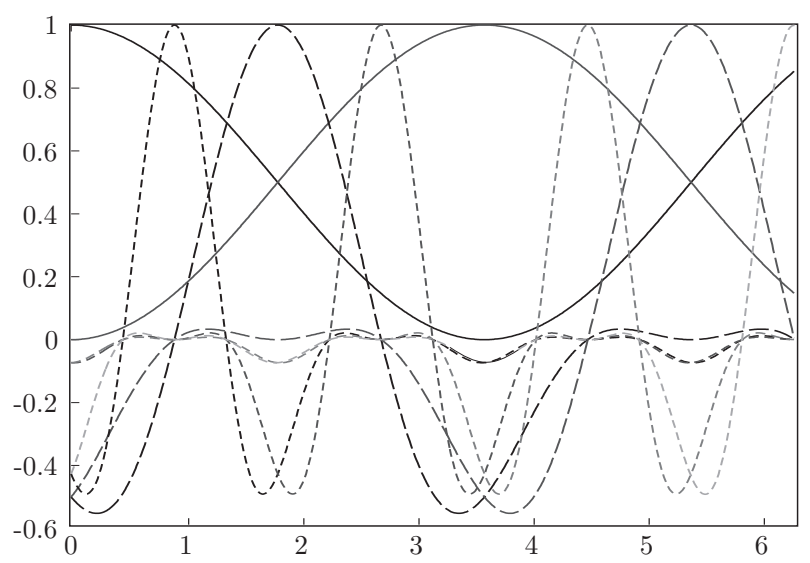

Fig. 3 Functions for decomposition in $V_{2}$.

\section{Governing equations of the beam}

The complete set of Reissner's beam equations [13] consists of the constitutive equations (27)-(28), the equilibrium equations (29)-(30) and the kinematic equations (31)-(32) (see [22], [23]):

$\boldsymbol{f}_{1}=\mathbf{R}(x) \mathscr{C}_{N}\left(\boldsymbol{\gamma}_{G}(x), \boldsymbol{\kappa}_{G}(x)\right)-\boldsymbol{N}_{g}(x)=\mathbf{0}$

$\boldsymbol{f}_{2}=\mathbf{R}(x) \mathscr{C}_{M}\left(\boldsymbol{\gamma}_{G}(x), \boldsymbol{\kappa}_{G}(x)\right)-\boldsymbol{M}_{g}(x)=\mathbf{0}$

$\boldsymbol{f}_{3}=\boldsymbol{N}_{g}^{\prime}(x)+\boldsymbol{n}_{g}(x)=\mathbf{0}$

$\boldsymbol{f}_{4}=\boldsymbol{M}_{g}^{\prime}(x)+\boldsymbol{m}_{g}(x)-\boldsymbol{N}_{g}(x) \times \boldsymbol{r}_{g}^{\prime}(x)=\mathbf{0}$

$\boldsymbol{f}_{5}=\boldsymbol{r}_{g}^{\prime}(x)-\mathbf{R}(x)\left(\boldsymbol{\gamma}_{G}(x)-\boldsymbol{c}_{G}(x)\right)=\mathbf{0}$

$\boldsymbol{f}_{6}=\boldsymbol{\vartheta}_{g}^{\prime}(x)-\mathbf{T}^{-T}(x)\left(\boldsymbol{\kappa}_{G}(x)-\boldsymbol{d}_{G}(x)\right)=\mathbf{0}$.

The related static boundary conditions are

$\boldsymbol{F}^{0}+\boldsymbol{N}_{g}(0)=\mathbf{0}$

$\boldsymbol{P}^{0}+\boldsymbol{M}_{g}(0)=\mathbf{0}$

$\boldsymbol{F}^{L}-\boldsymbol{N}_{g}(L)=\mathbf{0}$

$\boldsymbol{P}^{L}-\boldsymbol{M}_{g}(L)=\mathbf{0}$. 


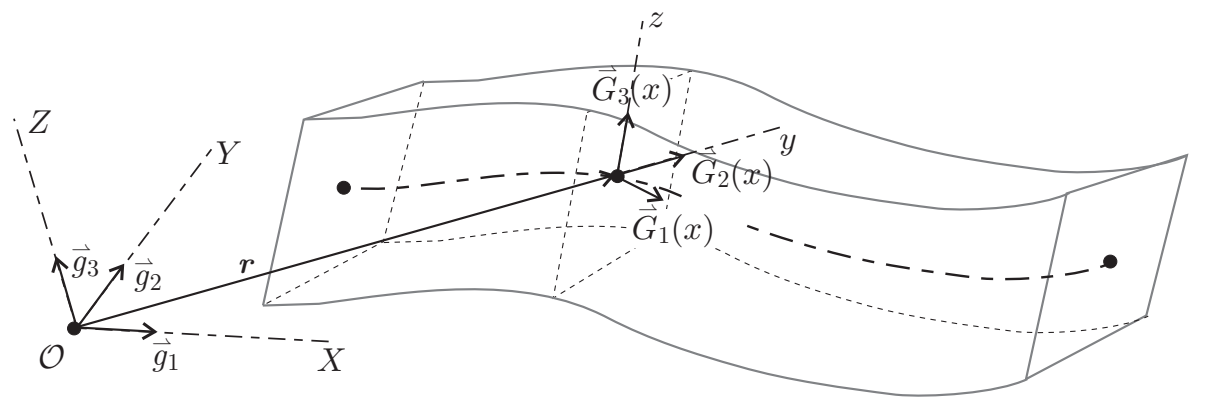

Fig. 4 Model of the three-dimensional beam.

Here, the prime $\left({ }^{\prime}\right)$ denotes the derivative with respect to the arc-length parameter of the reference line in the initial configuration, $x$, and " $\times$ " marks the cross vector product. The meaning of the notations used in the above equations is described below (see also Figure 4):

$g$ fixed (inertial) orthonormal basis $\left\{\vec{g}_{1}, \vec{g}_{2}, \vec{g}_{3}\right\}$ spanning the physical space of the beam;

$G$ orthonormal basis $\left\{\vec{G}_{1}, \vec{G}_{2}, \vec{G}_{3}\right\}$ spanning the cross-sectional planes;

$\boldsymbol{N}, \boldsymbol{M}$ stress-resultant force and moment vectors over the crosssection;

$\mathscr{C}_{N}, \mathscr{C}_{M}$ operators describing material of the beam;

$\gamma$ translational strain vector $\left(\gamma_{G 1}\right.$ is the extensional strain, $\boldsymbol{\gamma}_{G 2}, \boldsymbol{\gamma}_{G 3}$ are shear strains);

$\boldsymbol{\kappa}$ rotational strain vector ( $\kappa_{G 1}$ is the torsional strain, $\kappa_{G 2}$, $\kappa_{G 3}$ are the curvatures);

$\boldsymbol{r}$ position vector of the reference line of the beam;

$\mathbf{R}$ both rotation matrix from $g$ to $G$ (i.e., $\mathbf{R}=\mathbf{I}+\frac{\sin \vartheta}{\vartheta} \boldsymbol{\Theta}+$ $\left.\frac{1-\cos \vartheta}{\vartheta^{2}} \boldsymbol{\Theta}^{2}, \vartheta=\left\|\boldsymbol{\vartheta}_{g}\right\|\right)$ and coordinate transformation ma$\operatorname{trix}\left(\boldsymbol{v}_{g}=\mathbf{R} \boldsymbol{v}_{G}\right)$;

$\vartheta$ rotational vector whose axis coincides with the axis of rotation and whose length equals the angle of rotation;

$\boldsymbol{\Theta}$ skew-symmetric matrix $\boldsymbol{\Theta}$ composed from its axial vector $\boldsymbol{\vartheta}_{g}=\left[\begin{array}{lll}\vartheta_{g 1} & \vartheta_{g 2} & \vartheta_{g 3}\end{array}\right]^{T}$

$\mathbf{T}^{T}$ transformation matrix between $\boldsymbol{\kappa}_{G}$ and $\boldsymbol{\vartheta}_{g}^{\prime}$ (i.e., $\mathbf{T}^{T}=$ $\left.\mathbf{I}-\frac{1-\cos \vartheta}{\vartheta^{2}} \boldsymbol{\Theta}+\frac{\vartheta-\sin \vartheta}{\vartheta^{3}} \boldsymbol{\Theta}^{2}, \vartheta=\left\|\boldsymbol{\vartheta}_{g}\right\|\right)$;

$\boldsymbol{c}, \boldsymbol{d}$ variational constants determined from the known strains, position vectors and rotations in the initial configuration;

$\boldsymbol{n}, \boldsymbol{m}$ external distributed force and moment vectors per unit of the undeformed length of the axis;

$\boldsymbol{F}^{0}, \boldsymbol{F}^{L}$ external point forces at the boundaries $x=0, x=L$;

$\boldsymbol{P}^{0}, \boldsymbol{P}^{L}$ external point moments at the boundaries $x=0, x=L$;

$\bullet^{0}, \bullet^{L}$ discrete value of a quantity at the boundaries $x=0, x=$ $L$.

It is found useful to replace the cross vector product by the adequate matrix product. After we introduce the skewsymmetric matrix $\mathbf{S}(\boldsymbol{v})$ formed from the components of vec- tor $\boldsymbol{v}$, the cross vector product $\boldsymbol{v} \times \boldsymbol{u}$ can be written as:

$\boldsymbol{v} \times \boldsymbol{u}=\left[\begin{array}{l}v_{2} u_{3}-v_{3} u_{2} \\ v_{3} u_{1}-v_{1} u_{3} \\ v_{1} u_{2}-v_{2} u_{1}\end{array}\right]=\left[\begin{array}{ccc}0 & -v_{3} & v_{2} \\ v_{3} & 0 & -v_{1} \\ -v_{2} & v_{1} & 0\end{array}\right]\left[\begin{array}{l}u_{1} \\ u_{2} \\ u_{3}\end{array}\right]=\mathbf{S}(\boldsymbol{v}) \boldsymbol{u}$.

\section{Linearized equations of the beam}

\subsection{Mathematical background}

Mathematically, equations (27)-(32) introduce 24 scalar functionals, dependent on the (primary) unknowns $\boldsymbol{r}_{g}(x), \boldsymbol{\vartheta}_{g}(x)$, $\boldsymbol{N}_{g}(x), \boldsymbol{M}_{g}(x), \boldsymbol{\gamma}_{G}(x)$, and $\boldsymbol{\kappa}_{G}(x)$. Let $\boldsymbol{y}=\left(y_{1}, y_{2}, \ldots, y_{n}\right)$ denote the entire vector of unknowns. The linearization of the functional $\mathscr{F}(\boldsymbol{y})$ about an arbitrary value $\boldsymbol{y}=\boldsymbol{y}_{0}$ is defined by

$$
\delta \mathscr{F}\left(\boldsymbol{y}_{0} ; \boldsymbol{y} \boldsymbol{y}\right)=-\mathscr{F}\left(\boldsymbol{y}_{0}\right),
$$

where $\delta \mathscr{F}$ denotes the variation of the functional at $\boldsymbol{y}_{0}$ in the direction $\delta y$

$\boldsymbol{\delta} \mathscr{F}\left(\boldsymbol{y}_{0} ; \boldsymbol{y}\right)=\left.\sum_{j=1}^{n} \frac{\partial \mathscr{F}}{\partial y_{j}}\right|_{\boldsymbol{y}_{0}} \delta y_{j}$.

This equation holds in the linear vector spaces.

The linearization of a functional in non-linear spaces requires a different procedure. In our case the rotation matrix $\mathbf{R}$ is expressed in terms of the non-additive rotational vector $\boldsymbol{\vartheta}_{g}$ (see [1], [6] for further details). If the change of $\boldsymbol{\vartheta}_{g}$ is denoted by $\alpha \delta \vartheta_{g}$, the rotational operator at the perturbed value of its argument is the product $\mathbf{R}\left(\alpha \delta \boldsymbol{\vartheta}_{g}\right) \mathbf{R}\left(\boldsymbol{\vartheta}_{g}\right)$, so that the difference between the original and the perturbed value of the rotational operator is

$\Delta \mathbf{R}=\mathbf{R}\left(\alpha \delta \boldsymbol{\vartheta}_{g}\right) \mathbf{R}\left(\boldsymbol{\vartheta}_{g}\right)-\mathbf{R}\left(\boldsymbol{\vartheta}_{g}\right)$.

The Gâteaux variation of $\mathbf{R}$ then follows from the definition [19]:

$$
\begin{aligned}
\delta \mathbf{R} & =\left.\frac{d}{d \alpha}\right|_{\alpha=0} \mathbf{R}\left(\alpha \delta \boldsymbol{\vartheta}_{g}\right) \mathbf{R}\left(\boldsymbol{\vartheta}_{g}\right) \\
& =\delta \Theta \mathbf{R},
\end{aligned}
$$


where $\delta \boldsymbol{\theta}$ means the skew-symmetric matrix, whose components are formed from its axial vector $\delta \boldsymbol{\vartheta}_{g}$.

When multiplied by an arbitrary vector, $\boldsymbol{u}$, equation (42) gives

$\delta \mathbf{R} \boldsymbol{u}=\delta \boldsymbol{\Theta R} \boldsymbol{u}=\mathbf{S}\left(\boldsymbol{\delta} \boldsymbol{\vartheta}_{g}\right) \mathbf{R} \boldsymbol{u}=\delta \boldsymbol{\vartheta}_{g} \times \mathbf{R} \boldsymbol{u}$

Taking into account that $\boldsymbol{v} \times \boldsymbol{u}=-\boldsymbol{u} \times \boldsymbol{v}$ and considering (37) we get

$\delta \mathbf{R} u=\delta \boldsymbol{\vartheta}_{g} \times \mathbf{R} u=-\mathbf{R} u \times \delta \boldsymbol{\vartheta}_{g}=-\mathbf{S}(\mathbf{R} u) \delta \boldsymbol{\vartheta}_{g}$

\subsection{Linearization of equations of the beam}

The linearization of the constitutive equations gives:

$\delta \mathscr{C}_{N}=\mathrm{C}_{\gamma \gamma} \delta \gamma_{G}+\mathrm{C}_{\gamma \kappa} \delta \kappa_{G}$

$\delta \mathscr{C}_{M}=\mathrm{C}_{\kappa \gamma} \delta \boldsymbol{\gamma}_{G}+\mathrm{C}_{\kappa \kappa} \delta \boldsymbol{\kappa}_{G}$.

Here the components of matrices $\mathbf{C}_{\gamma \gamma}, \mathbf{C}_{\gamma \kappa}, \mathbf{C}_{\kappa \gamma}$, and $\mathbf{C}_{\kappa \kappa}$ are the partial derivatives of $\mathscr{C}_{N}$ and $\mathscr{C}_{M}$ with respect to the components of $\boldsymbol{\gamma}_{G}$ and $\boldsymbol{\kappa}_{G}$ :

$\begin{array}{ll}\mathbf{C}_{\gamma \gamma}=\left[\frac{\partial \mathscr{C}_{i}^{N}}{\partial \gamma_{j}}\right], & \mathbf{C}_{\gamma \kappa}=\left[\frac{\partial \mathscr{C}_{i}^{N}}{\partial \kappa_{j}}\right] \\ \mathbf{C}_{\kappa \gamma}=\left[\frac{\partial \mathscr{C}_{i}^{M}}{\partial \gamma_{j}}\right], & \mathbf{C}_{\kappa \kappa}=\left[\frac{\partial \mathscr{C}_{i}^{M}}{\partial \kappa_{j}}\right] .\end{array}$

The matrix $\mathbf{C}=\left[\begin{array}{ll}\mathbf{C}_{\gamma \gamma} & \mathbf{C}_{\gamma \kappa} \\ \mathbf{C}_{\kappa \gamma} & \mathbf{C}_{\kappa \kappa}\end{array}\right]$ is called the cross-section constitutive tangent matrix.

After equation (31) is inserted in (30) and equations (44)(45) taken into account, the variations of the equations of the beam are easily derived and are as follows:

$$
\begin{aligned}
\delta \boldsymbol{f}_{1} & =\delta \Theta \mathrm{R} \mathscr{C}_{N}+\mathrm{RC} C_{\gamma \gamma} \delta \boldsymbol{\gamma}_{G}+\mathrm{RC}_{\gamma \kappa} \delta \boldsymbol{\kappa}_{G}-\delta \boldsymbol{N}_{g} \\
\delta \boldsymbol{f}_{2} & =\delta \Theta \mathrm{R} \mathscr{C}_{M}+\mathrm{RC}_{\kappa \gamma} \delta \boldsymbol{\gamma}_{G}+\mathrm{RC}_{\kappa \kappa} \delta \boldsymbol{\kappa}_{G}-\delta \boldsymbol{M}_{g} \\
\delta \boldsymbol{f}_{3} & =\delta \boldsymbol{N}_{g}^{\prime} \\
\delta \boldsymbol{f}_{4} & =\delta \boldsymbol{M}_{g}^{\prime}-\delta \boldsymbol{N}_{g} \times \boldsymbol{r}_{g}^{\prime}-\boldsymbol{N}_{g} \times \delta \boldsymbol{r}_{g}^{\prime} \\
\delta \boldsymbol{f}_{5} & =\delta \boldsymbol{r}_{g}^{\prime}-\delta \Theta \mathrm{R}\left(\boldsymbol{\gamma}_{G}-\boldsymbol{c}_{G}\right)-\mathrm{R} \delta \boldsymbol{\gamma}_{G} \\
\delta \boldsymbol{f}_{6} & =\delta \boldsymbol{\vartheta}_{g}^{\prime}-\mathrm{R} \delta \boldsymbol{\kappa}_{G} .
\end{aligned}
$$

Here, it is assumed that $\boldsymbol{n}_{g}(x)$ and $\boldsymbol{m}_{g}(x)$ do not depend on the unknown functions. Note that the derivation of equation (51) from (32) is not straightforward. The proof can be found in [6] or [22].

We assume that the geometry of the beam and its strains in the initial configuration $\boldsymbol{y}_{0}$ are arbitrary, yet kinematically exact, so that equations (31)-(32) are identically satisfied (i.e., $\left.\boldsymbol{f}_{5}\left(\boldsymbol{y}_{0}\right)=\boldsymbol{f}_{6}\left(\boldsymbol{y}_{0}\right)=\mathbf{0}\right)$. Such a beam is called a naturally strained (extended, curved and twisted) beam. We also assume that, in the initial configuration, stress-resultant vectors are identically zero, $\boldsymbol{N}_{g}(x)=\boldsymbol{M}_{g}(x)=\mathbf{0}$. The set of linearized equations then takes the form

$$
\begin{aligned}
& \delta \Theta \mathrm{R} \mathscr{C}_{N}+\mathrm{RC}_{\gamma \gamma} \delta \boldsymbol{\gamma}_{G}+\mathrm{RC}_{\gamma \kappa} \delta \boldsymbol{\kappa}_{G}-\delta \boldsymbol{N}_{g}=-\mathrm{R} \mathscr{C}_{N} \\
& \delta \Theta \mathrm{R} \mathscr{C}_{M}+\mathrm{RC}_{\kappa \gamma} \delta \boldsymbol{\gamma}_{G}+\mathrm{RC}_{\kappa \kappa} \delta \boldsymbol{\kappa}_{G}-\delta \boldsymbol{M}_{g}=-\mathrm{R} \mathscr{C}_{M} \\
& \delta \boldsymbol{N}_{g}^{\prime}=-\boldsymbol{n}_{g} \\
& \delta \boldsymbol{M}_{g}^{\prime}-\delta \boldsymbol{N}_{g} \times \boldsymbol{r}_{g}^{\prime}=-\boldsymbol{m}_{g} \\
& \delta \boldsymbol{r}_{g}^{\prime}-\delta \Theta \mathrm{R}\left(\boldsymbol{\gamma}_{G}-\boldsymbol{c}_{G}\right)-\mathrm{R} \delta \boldsymbol{\gamma}_{G}=0 \\
& \delta \boldsymbol{\vartheta}_{g}^{\prime}-\mathrm{R} \delta \boldsymbol{\kappa}_{G}=0 .
\end{aligned}
$$

All the quantities are dependent on parameter $x$, which was left out from equations for the sake of clearness. The linearized form of static boundary conditions reads

$$
\begin{aligned}
& \delta \boldsymbol{N}_{g}^{0}=-\boldsymbol{F}^{0} \\
& \delta \boldsymbol{M}_{g}^{0}=-\boldsymbol{P}^{0} \\
& \delta \boldsymbol{N}_{g}(L)=\boldsymbol{F}^{L} \\
& \delta \boldsymbol{M}_{g}(L)=\boldsymbol{P}^{L} .
\end{aligned}
$$

The set of equations (52)-(61) can further be reduced by integrating equations (54)-(57). By rearranging the terms in (56)-(57) and considering (31) and (43) we obtain

$$
\begin{aligned}
\delta \boldsymbol{r}_{g} & =\delta \boldsymbol{r}_{g}^{0}+\int_{0}^{x}\left\{-\mathbf{S}\left(\mathbf{R} \boldsymbol{r}_{g}^{\prime}\right) \boldsymbol{\delta} \boldsymbol{\vartheta}_{g}+\mathbf{R} \boldsymbol{\delta} \boldsymbol{\gamma}_{G}\right\} d \xi \\
\delta \boldsymbol{\vartheta}_{g} & =\delta \boldsymbol{\vartheta}_{g}^{0}+\int_{0}^{x} \mathbf{R} \delta \boldsymbol{\kappa}_{G} d \xi .
\end{aligned}
$$

Inserting (62) into (63) yields

$$
\begin{aligned}
\boldsymbol{\delta} \boldsymbol{r}_{g} & =\delta \boldsymbol{r}_{g}^{0}-\int_{0}^{x} \mathbf{S}\left(\mathbf{R} \boldsymbol{r}_{g}^{\prime}\right) d \xi \delta \boldsymbol{\vartheta}_{g}^{0} \\
& +\int_{0}^{x}\left\{-\mathbf{S}\left(\mathbf{R} \boldsymbol{r}_{g}^{\prime}\right) \mathbf{R} \boldsymbol{\delta} \boldsymbol{\kappa}_{G}+\mathbf{R} \boldsymbol{\delta} \boldsymbol{\gamma}_{G}\right\} d \xi .
\end{aligned}
$$

Integration of (54)-(55) with consideration of (37) results in

$$
\begin{aligned}
\delta \boldsymbol{N}_{g}(x)= & \delta \boldsymbol{N}_{g}^{0}-\int_{0}^{x} \boldsymbol{n}_{g} d \xi \\
\delta \boldsymbol{M}_{g}(x)= & \delta \boldsymbol{M}_{g}^{0}+\int_{0}^{x} \delta \boldsymbol{N}_{g} \times \boldsymbol{r}_{g}^{\prime} d \xi-\int_{0}^{x} \boldsymbol{m}_{g} d \xi \\
= & \delta \boldsymbol{M}_{g}^{0}-\mathbf{S}\left(\int_{0}^{x} \boldsymbol{r}_{g}^{\prime} d \xi\right) \delta \boldsymbol{N}_{g}^{0} \\
& +\int_{0}^{x} \mathbf{S}\left(\boldsymbol{r}_{g}^{\prime}\right)\left(\int_{0}^{\xi} \boldsymbol{n}_{g} d \eta\right) d \xi-\int_{0}^{x} \boldsymbol{m}_{g} d \boldsymbol{\xi} \\
= & \delta \boldsymbol{M}_{g}^{0}-\mathbf{S}\left(\boldsymbol{r}_{g}(x)-\boldsymbol{r}_{g}^{0}\right) \delta \boldsymbol{N}_{g}^{0} \\
& +\int_{0}^{x} \mathbf{S}\left(\boldsymbol{r}_{g}^{\prime}\right)\left(\int_{0}^{\xi} \boldsymbol{n}_{g} d \eta\right) d \xi-\int_{0}^{x} \boldsymbol{m}_{g} d \xi .
\end{aligned}
$$

The final form of the linearized equations of three-dimensional beam then reads 


$$
\begin{array}{r}
-\mathbf{S}\left(\mathbf{R} \mathscr{C}_{N}\right) \delta \boldsymbol{\vartheta}_{g}^{0}-\mathbf{S}\left(\mathbf{R} \mathscr{C}_{N}\right) \int_{0}^{x} \mathbf{R} \delta \boldsymbol{\kappa}_{G} d \xi+\mathbf{R} \boldsymbol{C}_{\gamma \gamma} \delta \boldsymbol{\gamma}_{G} \\
\quad+\mathbf{R} \boldsymbol{C}_{\gamma \kappa} \delta \boldsymbol{\kappa}_{G}-\delta \boldsymbol{N}_{g}^{0}=-\mathbf{R} \mathscr{C}_{N}-\int_{0}^{x} \boldsymbol{n}_{g} d \xi \\
-\mathbf{S}\left(\mathbf{R} \mathscr{C}_{M}\right) \delta \boldsymbol{\vartheta}_{g}^{0}-\mathbf{S}\left(\mathbf{R} \mathscr{C}_{M}\right) \int_{0}^{x} \mathbf{R} \delta \boldsymbol{\kappa}_{G} d \xi+\mathbf{R} \boldsymbol{C}_{\kappa \gamma} \delta \boldsymbol{\gamma}_{G} \\
+\mathbf{R} \boldsymbol{C}_{\kappa \kappa} \delta \boldsymbol{\kappa}_{G}-\delta \boldsymbol{M}_{g}^{0}=-\mathbf{R} \mathscr{C}_{M} \\
\quad-\int_{0}^{x} \boldsymbol{m}_{g} d \xi+\int_{0}^{x} \mathbf{S}\left(\boldsymbol{r}_{g}^{\prime}\right)\left(\int_{0}^{\xi} \boldsymbol{n}_{g} d \eta\right) d \xi \\
\delta \boldsymbol{r}_{g}^{L}-\delta \boldsymbol{r}_{g}^{0}+\int_{0}^{L} \mathbf{S}\left(\mathbf{R} \boldsymbol{r}_{g}^{\prime}\right) d \xi \delta \boldsymbol{\vartheta}_{g}^{0} \\
\quad-\int_{0}^{L}\left\{-\mathbf{S}\left(\mathbf{R} \boldsymbol{r}_{g}^{\prime}\right) \mathbf{R} \delta \boldsymbol{\kappa}_{G}+\mathbf{R} \delta \boldsymbol{\gamma}_{G}\right\} d x=0 \\
\delta \boldsymbol{\vartheta}_{g}^{L}-\delta \boldsymbol{\vartheta}_{g}^{0}-\int_{0}^{L} \mathbf{R} \delta \boldsymbol{\kappa}_{G} d x=0 \\
\delta \boldsymbol{N}_{g}^{0}=-\boldsymbol{F}^{0} \\
\delta \boldsymbol{M}_{g}^{0}=-\boldsymbol{P}^{0} \\
\delta \boldsymbol{N}_{g}^{0}=\boldsymbol{F}^{L}+\int_{0}^{L} \boldsymbol{n}_{g} d x \\
\delta \boldsymbol{M}_{g}^{0}-\mathbf{S}\left(\boldsymbol{r}_{g}^{L}-\boldsymbol{r}_{g}^{0}\right) \delta \boldsymbol{N}_{g}^{0}=\boldsymbol{P}^{L}+\int_{0}^{L} \boldsymbol{m}_{g} d x \\
\quad-\int_{0}^{L} \mathbf{S}\left(\boldsymbol{r}_{g}^{\prime}\right)\left(\int_{0}^{x} \boldsymbol{n}_{g} d \xi\right) d x .
\end{array}
$$

Equations (67)-(74) represent a system of linear equations for two unknown functions $\delta \boldsymbol{\gamma}_{G}(x)$ and $\delta \boldsymbol{\kappa}_{G}(x)$, boundary kinematic vectors $\delta \boldsymbol{r}_{g}^{0}, \delta \boldsymbol{\vartheta}_{g}^{0}, \delta \boldsymbol{r}_{g}^{L}, \delta \boldsymbol{\vartheta}_{g}^{L}$, and boundary equilibrium stress resultants $\delta \boldsymbol{N}_{g}^{0}, \delta \boldsymbol{M}_{g}^{0}$. The remaining quantities are known from the initial configuration of the beam.

\section{Numerical solution of linearized equations}

Equations (67)-(74) are generally too demanding to be solved analytically. That is why discretization of equations and interpolation of unknown functions need to be introduced.

Unknown functions $\delta \boldsymbol{\gamma}_{G}(x)$ and $\delta \boldsymbol{\kappa}_{G}(x)$ are replaced by the set of their unknown values $\delta \boldsymbol{\gamma}_{G}^{p}$ and $\delta \boldsymbol{\kappa}_{G}^{p}$ at discrete points identified by $x_{p} \in[0, L] ; p=0,1, \ldots, 2^{j+1}-1,(j \geq$ $0)$ and interpolated by the set of $2^{j+1}$ interpolation functions $I_{p}(x)$

$$
\begin{gathered}
\delta \boldsymbol{\gamma}_{G}(x)=\sum_{p=0}^{2^{j+1}-1} I_{p}(x) \delta \boldsymbol{\gamma}_{G}^{p} \\
\delta \boldsymbol{\kappa}_{G}(x)=\sum_{p=0}^{2^{j+1}-1} I_{p}(x) \delta \boldsymbol{\kappa}_{G}^{p}
\end{gathered}
$$

Several options will be tested for interpolation functions: (i) scaling functions (1), (ii) wavelet functions (8), and (iii) the combination of scaling and wavelet functions with respect to the decomposition (19).

The discretization of equations (67)-(68) is achieved by satisfying them at $2^{j+1}$ pre-selected discrete points $x_{q} \in[0, L]$; $q=1, \ldots, 2^{j+1}$, not necessarily coincidental with any of the interpolation points. In the present paper, $2^{j+1}$ Gaussian points are chosen as suitable collocation points for (67)(68). Note that the Gaussian points are chosen for both discretization and numerical integration, which is suitable for the implementation of the numerical integrals in the algorithm. The interpolation and discretization lead to $2^{j+1}+6$ algebraic equations for $2^{j+1}+6$ unknown vectors $\boldsymbol{r}_{g}^{0}, \boldsymbol{\vartheta}_{g}^{0}$, $\boldsymbol{N}_{g}^{0}, \boldsymbol{M}_{g}^{0}, \boldsymbol{r}_{g}^{L}, \boldsymbol{\vartheta}_{g}^{L}, \boldsymbol{\gamma}_{G}^{p}$, and $\boldsymbol{\kappa}_{G}^{p}, p=0, \ldots, 2^{j+1}-1$ :

$$
\begin{gathered}
-\mathbf{S}\left(\mathbf{R} \mathscr{C}_{N}\right) \delta \boldsymbol{\vartheta}_{g}^{0}-\delta \boldsymbol{N}_{g}^{0}+\sum_{p=0}^{2^{j+1}-1} \mathbf{R}^{q} \mathbf{C}_{\gamma \gamma}^{q} I_{p}^{q} \delta \boldsymbol{\gamma}_{G}^{p} \\
+\sum_{p=0}^{2^{j+1}-1}\left\{-\mathbf{S}\left(\mathbf{R}^{q} \mathscr{C}_{N}^{q}\right) \int_{0}^{x_{q}} \mathbf{R} I_{p} d \xi+\mathbf{R}^{q} \mathbf{C}_{\gamma \kappa}^{q} I_{p}^{q}\right\} \delta \boldsymbol{\kappa}_{G}^{p} \\
=-\mathbf{R} \mathscr{C}_{N}\left(x_{q}\right)-\int_{0}^{x_{q}} \boldsymbol{n}_{g} d \xi
\end{gathered}
$$

$$
\begin{gathered}
-\mathbf{S}\left(\mathbf{R} \mathscr{C}_{M}\right) \delta \boldsymbol{\vartheta}_{g}^{0}+\mathbf{S}\left(\boldsymbol{r}_{g}\left(x_{q}\right)-\boldsymbol{r}_{g}^{0}\right) \delta \boldsymbol{N}_{g}^{0}+\sum_{p=0}^{2^{j+1}-1} \mathbf{R}^{q} \mathbf{C}_{\kappa \gamma}^{q} I_{p}^{q} \delta \boldsymbol{\gamma}_{G}^{p} \\
-\delta \boldsymbol{M}_{g}^{0}+\sum_{p=0}^{2^{j+1}-1}\left\{-\mathbf{S}\left(\mathbf{R}^{q} \mathscr{C}_{M}^{q}\right) \int_{0}^{x_{q}} \mathbf{R} I_{p} d \xi+\mathbf{R}^{q} \mathbf{C}_{\kappa \kappa}^{q} I_{p}^{q}\right\} \delta \boldsymbol{\kappa}_{G}^{p} \\
=-\mathbf{R} \mathscr{C}_{M}\left(x_{q}\right)-\int_{0}^{x_{q}} \boldsymbol{m}_{g} d \xi \\
+\int_{0}^{x_{q}} \mathbf{S}\left(\boldsymbol{r}_{g}^{\prime}\right)\left(\int_{0}^{\xi} \boldsymbol{n}_{g} d \eta\right) d \xi
\end{gathered}
$$

$\delta \boldsymbol{r}_{g}^{L}-\delta \boldsymbol{r}_{g}^{0}+\int_{0}^{L} \mathbf{S}\left(\mathbf{R} \boldsymbol{r}_{g}^{\prime}\right) d x \delta \boldsymbol{\vartheta}_{g}^{0}-\sum_{p=0}^{2^{j+1}-1} \int_{0}^{L} \mathbf{R} \boldsymbol{I}_{p} d x \delta \boldsymbol{\gamma}_{G}^{p}$

$$
+\sum_{p=0}^{2^{j+1}-1} \int_{0}^{L}-\mathbf{S}\left(\mathbf{R} \boldsymbol{r}_{g}^{\prime}\right) \mathbf{R} d x \delta \boldsymbol{\kappa}_{G}^{p}=0
$$

$$
\delta \boldsymbol{\vartheta}_{g}^{L}-\delta \boldsymbol{\vartheta}_{g}^{0}-\sum_{p=0}^{2^{j+1}-1} \int_{0}^{L} \mathbf{R} I_{p} d x \delta \boldsymbol{\kappa}_{G}^{p}=0
$$

$\delta \boldsymbol{N}_{g}^{0}=-\boldsymbol{F}^{0}$

$\delta \boldsymbol{M}_{g}^{0}=-\boldsymbol{P}^{0}$

$\delta \boldsymbol{N}_{g}^{0}=\boldsymbol{F}^{L}+\int_{0}^{L} \boldsymbol{n}_{g} d x$

$$
\begin{gathered}
\delta \boldsymbol{M}_{g}^{0}-\mathbf{S}\left(\boldsymbol{r}_{g}^{L}-\boldsymbol{r}_{g}^{0}\right) \delta \boldsymbol{N}_{g}^{0}=\boldsymbol{P}^{L}+\int_{0}^{L} \boldsymbol{m}_{g} d x \\
-\int_{0}^{L} \mathbf{S}\left(\boldsymbol{r}_{g}^{\prime}\right)\left(\int_{0}^{x} \boldsymbol{n}_{g} d \xi\right) d x .
\end{gathered}
$$

The upper index $q$ denotes the discrete value of a quantity at the discretization point $x_{q}$. The system of equations (77)-(84) is linear algebraic with known coefficients, thus it is easy to be solved.

\section{Numerical examples}

We present several numerical examples in order to demonstrate the accuracy and stability and compare the wavelet 
beam formulation to various polynomial formulations. In accord with the theoretical assumption arbitrary finite initial bending and/or twisting curvature of the beam can be prescribed at the unloaded initial configuration, but only a small loading is allowed to be applied in order to achieve small displacements, rotations and strains. In order to present the full benefit of the wavelet-based discretization, we take only a low number of elements in the mesh, while simultaneously employing elements of high orders. The rate of an element is described by $j$; then the number of interpolation points is $2^{j+1}$. For each $j$, the results, obtained by Lagrangian interpolation functions, scaling interpolation functions, and wavelet-based decomposition functions are presented.

The operators $\mathscr{C}_{N}$ and $\mathscr{C}_{M}$ in (27)-(28) are taken to be linear, so that the cross-section constitutive tangent matrix reads

$\mathbf{C}=\left[\begin{array}{cccccc}E A_{x} & 0 & 0 & 0 & E S_{y} & -E S_{z} \\ 0 & G A_{y} & 0 & 0 & 0 & 0 \\ 0 & 0 & G A_{z} & 0 & 0 & 0 \\ 0 & 0 & 0 & G J_{t} & 0 & 0 \\ E S_{y} & 0 & 0 & 0 & E J_{y} & E J_{y z} \\ -E S_{z} & 0 & 0 & 0 & E J_{y z} & E J_{z}\end{array}\right]$

$E$ and $G$ denote elastic and shear moduli of material; $A_{x}$ is the cross-sectional area; $J_{t}$ is the torsional inertial moment of the cross-section; $A_{y}$ and $A_{z}$ are the effective shear areas in directions $\vec{G}_{2}$ and $\vec{G}_{3}$ of the cross-section; $S_{y}$ and $S_{z}$ are the static moments of the cross-section about its directions $G_{2}$ and $G_{3} J_{y}$ and $J_{z}$ are the bending inertial moments of the cross-section about $\vec{G}_{2}$ and $\vec{G}_{3} ; J_{y z}$ is the product moment of inertia of the cross-section. Please note that in the beam model the choices of the parameters in cross-section constitutive tangent matrix are arbitrary quantities. It has been, however, observed by many researchers that the values of shear areas and torsional moment of cross-section can considerably affect the results. For the method of evaluation the torsional inertial moment and the shear areas in the case of an arbitrary cross section the reader is referred to the papers by Sapountzakis and Mokos [14], [15].

\subsection{Cantilever beam}

We consider a straight in-plane cantilever, subjected to a point moment at its free end (Figure 5). The geometric and material properties are as follows:

$$
\begin{aligned}
E & =2.1 \cdot 10^{4} \quad G=1.05 \cdot 10^{4} \quad L=100 \\
A_{x} & =20 \quad A_{y}=A_{z}=16 \\
J_{t} & =6.4566 \quad J_{y}=1.6667 \quad J_{z}=666.66 \\
S_{y} & =S_{z}=J_{y z}=0 .
\end{aligned}
$$

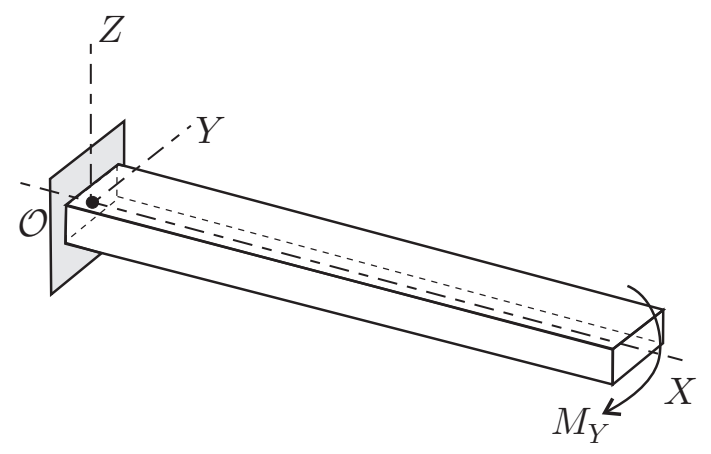

Fig. 5 The cantilever under free-end moment.

Table 1 Free-end displacement and rotation under an in-plane point moment; single element of order $2^{j+1}$.

\begin{tabular}{lrrr}
\hline \hline method & $j$ & $u_{Z}$ & $\vartheta_{Y}$ \\
\hline \hline Lagrange & 0 & 14.28571 & 0.28571 \\
Scaling & 0 & 14.28571 & 0.28571 \\
Decomposition & 0 & 14.28571 & 0.28571 \\
\hline Lagrange & 1 & 14.28571 & 0.28571 \\
Scaling & 1 & 14.28571 & 0.28571 \\
Decomposition & 1 & 13.57021 & 0.28571 \\
\hline Lagrange & 3 & 14.28571 & 0.28571 \\
Scaling & 3 & 14.28571 & 0.28571 \\
Decomposition & 3 & 14.15023 & 0.28571 \\
\hline Lagrange & 5 & 15.39210 & 0.28571 \\
Scaling & 5 & 14.28571 & 0.28571 \\
Decomposition & 5 & 14.27078 & 0.28571 \\
\hline Lagrange & 7 & divergence & divergence \\
Scaling & 7 & 14.28571 & 0.28571 \\
Decomposition & 7 & 14.28436 & 0.28571 \\
\hline \hline exact & & 14.28571 & 0.285714 \\
\hline \hline
\end{tabular}

The results for the free-end moment of magnitude $M_{Y}=100$ are shown in Table 1.

In Table 1 we compare the vertical displacement and the rotation at the free end; the exact linear solution is also displayed. Note that the problem is linear with respect to the free-end rotation, thus even the lowest order interpolation should give exact solution for the free-end rotation. This is confirmed in the last column of Table 1 . The vertical displacements, obtained by different approaches and different orders of interpolation, are quite accurate. We can observe that a high order Lagrangian interpolation is not a suitable choice. The relative error of Lagrangian approach for $j=5$ is about $8 \%$ for vertical displacement and the method is divergent for $j=7$. No such sensitivity can be observed for scaling functions and wavelet decomposition functions. A somewhat lower convergence rate of results is observed for the wavelet decomposition. 
6.2 Circular arch

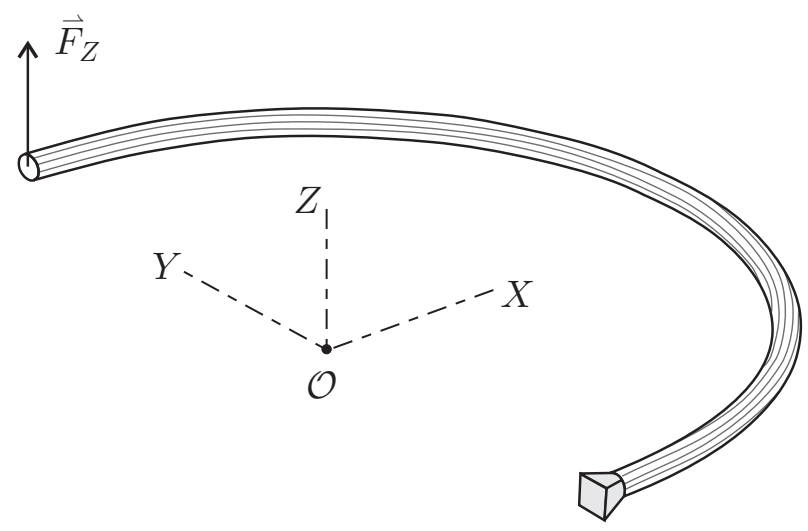

Fig. 6 Circular arch under in-plane and out-of-plane force.

Table 2 Out-of-plane force on circular arch; free-end displacements for a single element.

\begin{tabular}{lrrrr}
\hline \hline method & $j$ & $u_{Z}$ & $\Delta \vartheta_{X}$ & $\Delta \vartheta_{Y}$ \\
\hline \hline Lagrange & 0 & 8.396451 & 0.9425584 & 0.6406146 \\
Scaling & 0 & 8.746730 & 0.9425584 & 0.6406146 \\
Decomposition & 0 & 8.746730 & 0.9425584 & 0.6406146 \\
\hline Lagrange & 1 & 10.260494 & 0.9969752 & 0.6618478 \\
Scaling & 1 & 9.496759 & 0.9969752 & 0.6618478 \\
Decomposition & 1 & 11.715486 & 0.9969752 & 0.6618478 \\
\hline Lagrange & 3 & 10.132443 & 0.9969458 & 0.6618529 \\
Scaling & 3 & 9.906802 & 0.9969458 & 0.6618529 \\
Decomposition & 3 & 10.890450 & 0.9969458 & 0.6618529 \\
\hline Lagrange & 5 & 10.394379 & 0.996946 & 0.661853 \\
Scaling & 5 & 10.017767 & 0.9969458 & 0.6618529 \\
Decomposition & 5 & 10.511923 & 0.996946 & 0.661853 \\
\hline Lagrange & 7 & divergence & 0.996946 & 0.996946 \\
Scaling & 7 & 10.0579388 & 0.996946 & 0.661853 \\
Decomposition & 7 & 10.4302942 & 0.996946 & 0.661853 \\
\hline \hline Tabarrok et al. [16] & 9.931903 & 0.9931903 & 0.6618526 \\
\hline analytical [16] & 10.182905 & 0.996940 & 0.661850 \\
\hline \hline
\end{tabular}

We consider an elastic cantilever beam with the centroidal axis in the form of the circular arc with the central angle $\pi$ and radius $R=5$ (Figure 6). The cross-section of the beam is circular with radius $r=0.015$. Material moduli are $E=0.207 \cdot 10^{12}$ and $G=0.95 \cdot 10^{11}$. The cantilever is subjected to the vertical force $F_{Z}=100$ at the free end, which causes the out-of-plane deformation.

Results for a single element are shown in Table 2 and for a mesh of three elements in Table 3. Vertical displacements and rotations at the free end are presented. Present results are compared to theoretical and numerical results obtained by Tabarrok et al. [16]. The higher accuracy of rotations com- pared to the accuracy to displacements stems from simpler form of the analytical solution.

The present method gives excellent results also for a low number of elements or a lower order. By increasing the order of element we get more and more accurate results. This is, however, not the case in the Lagrangian polynomial-based formulation, where the divergence of higher order elements is observed. We should point out that the scaling functions and wavelet based formulations are stable for an arbitrary order of curved element.

Table 3 Out-of-plane force on circular arch; free-end displacements for three elements.

\begin{tabular}{|c|c|c|c|c|}
\hline method & $j$ & $u_{Z}$ & $\Delta \vartheta_{X}$ & $\Delta \vartheta_{Y}$ \\
\hline Lagrange & 0 & 10.111561 & 0.9965658 & 0.6614902 \\
\hline Scaling & 0 & 10.130604 & 0.9965658 & 0.6614902 \\
\hline Decomposition & 0 & 10.158515 & 0.9969458 & 0.6618529 \\
\hline Lagrange & 1 & 10.185741 & 0.9969458 & 0.6618529 \\
\hline Scaling & 1 & 10.150307 & 0.9969458 & 0.6618529 \\
\hline Decomposition & 1 & 10.138354 & 0.9969458 & 0.6618529 \\
\hline Lagrange & 3 & 10.182086 & 0.9969458 & 0.6618529 \\
\hline Scaling & 3 & 10.169346 & 0.9969458 & 0.6618529 \\
\hline Decomposition & 3 & 10.159792 & 0.9969458 & 0.6618529 \\
\hline Lagrange & 5 & 10.121157 & 0.9969458 & 0.6618529 \\
\hline Scaling & 5 & 10.176226 & 0.9969458 & 0.6618529 \\
\hline Decomposition & 5 & 10.158515 & 0.9969458 & 0.6618529 \\
\hline Lagrange & 7 & divergence & 0.9969458 & 0.6618529 \\
\hline Scaling & 7 & 10.1790165 & 0.996946 & 0.6618529 \\
\hline Decomposition & 7 & 10.155965 & 0.9969458 & 0.6618529 \\
\hline Tabarrok et al. [ & & 9.931903 & 0.9931903 & 0.6618526 \\
\hline analytical [16] & & 10.18290 & 0.996940 & 0.661850 \\
\hline
\end{tabular}

\subsection{Quarter-circular arch}

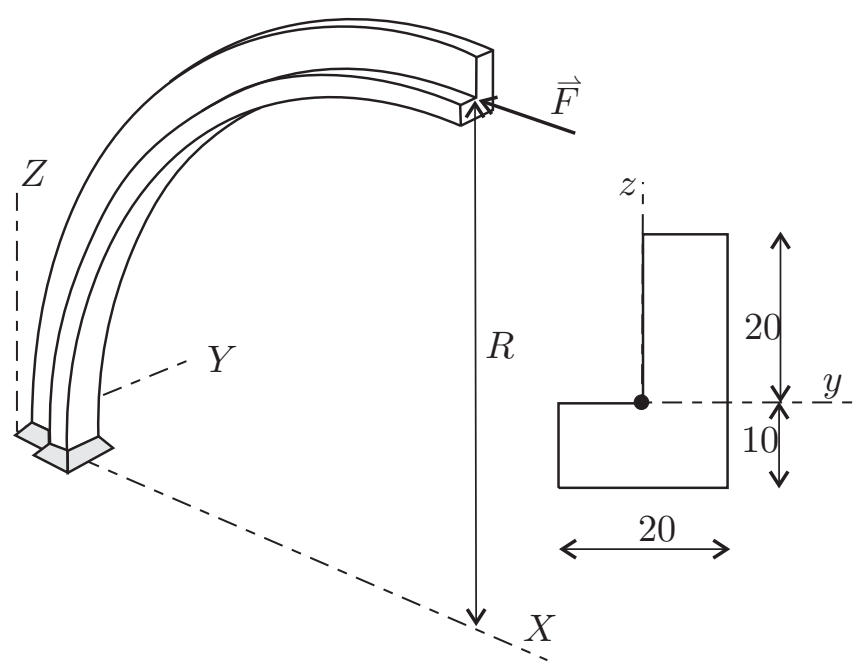

Fig. 7 Quarter-circular cantilever with non-symmetric cross-section. 
The horizontal force on circular cantilever arch with nonsymmetric cross-section results in spatial buckling of the arch. In this example we consider a quarter-circular cantilever with radius $R=250$ (Figure 7). The cross-section is non-symmetric; its geometry is given in Figure 7. Material moduli are $E=1000$ and $G=375.93$. The cantilever is subjected to the horizontal force $F=1$ at the free end, which causes the out-of-plane deformation.

Table 4 Spatial buckling of quarter-circular cantilever under free-end force; free-end displacements for three elements.

\begin{tabular}{lrrrr}
\hline \hline method & $j$ & $-u_{X}$ & $-u_{Y}$ & $u_{Z}$ \\
\hline \hline Lagrange & 0 & 0.302290 & 0.213697 & 0.213779 \\
Scaling & 0 & 0.303025 & 0.214610 & 0.214882 \\
Decomposition & 0 & 0.303025 & 0.214610 & 0.214882 \\
\hline Lagrange & 1 & 0.304560 & 0.216693 & 0.217553 \\
Scaling & 1 & 0.303741 & 0.215528 & 0.216029 \\
Decomposition & 1 & 0.313210 & 0.224808 & 0.225577 \\
\hline Lagrange & 3 & 0.306940 & 0.219320 & 0.220400 \\
Scaling & 3 & 0.306360 & 0.218589 & 0.219513 \\
Decomposition & 3 & 0.325670 & 0.237892 & 0.238542 \\
\hline Lagrange & 5 & 0.306845 & 0.221078 & 0.218052 \\
Scaling & 5 & 0.307203 & 0.219574 & 0.220635 \\
Decomposition & 5 & 0.326707 & 0.238933 & 0.239435 \\
\hline Lagrange & 7 & divergence & divergence & divergence \\
Scaling & 7 & 0.307466 & 0.219884 & 0.220993 \\
Decomposition & 7 & 0.326523 & 0.238713 & 0.239161 \\
\hline \hline 50 elements & & 0.305300 & 0.217503 & 0.218430 \\
\hline \hline
\end{tabular}

Displacements at the free end are shown in Table 4. The results for the mesh of three elements with various orders and interpolation types are compared to the results, obtained by the fine mesh of 50 polynomial cubic elements. The present formulations gives accurate results for low and moderate order elements with all types of interpolation. It should be pointed out the divergence of high order Lagrangian interpolation based elements, while scaling and wavelet based formulations show no lack of stability with order increase.

\subsection{Helical beam}

Our last example is the most demanding. The centroidal axis of the beam is taken in the shape of a helix. The radius of the helix is $R=5$ and the height is $H=5$ (Figure 8). Rotations about the vertical axis linearly increase from zero at the clamped point to $4 \pi$ at the free end, thus the projection of the axis to the plane describes two circles. The cross-section and the material properties are the same as in example 6.2. Unit concentrated force is applied in vertical direction at the free end.

Results for three and five finite-element meshes are shown in Tables 5 and 6. Numerical results are compared to the reference solution, obtained by the fine mesh of 50 polynomial

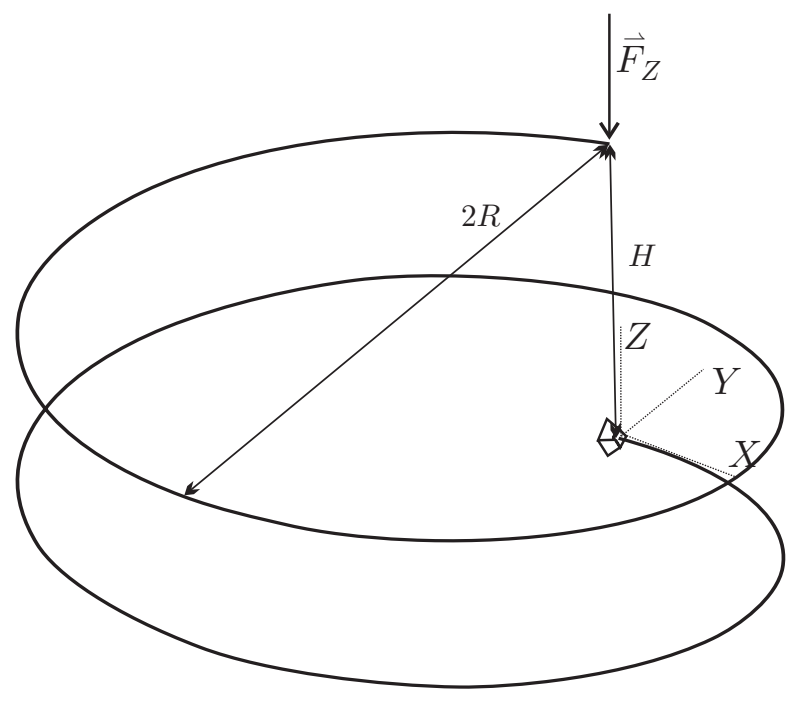

Fig. 8 Helical beam.

Table 5 Free-end displacements of helical beam; three finite elements.

\begin{tabular}{lrrrr}
\hline \hline method & $j$ & $u_{X}$ & $u_{Y}$ & $u_{Z}$ \\
\hline \hline Lagrange & 1 & 0.038808 & -0.118707 & -0.492068 \\
Scaling & 1 & 0.038254 & -0.127251 & -0.487350 \\
Decomposition & 1 & 0.026029 & -0.130552 & -0.508220 \\
\hline Lagrange & 3 & 0.030029 & -0.120985 & -0.487657 \\
Scaling & 3 & 0.030662 & -0.123197 & -0.486891 \\
Decomposition & 3 & 0.010961 & -0.151860 & -0.478188 \\
\hline Lagrange & 5 & -44.098029 & -2.287741 & 122.720285 \\
Scaling & 5 & 0.028863 & -0.122439 & -0.486349 \\
Decomposition & 5 & 0.011696 & -0.155967 & -0.474857 \\
\hline Lagrange & 6 & divergence & divergence & divergence \\
Scaling & 6 & 0.028592 & -0.122280 & -0.486249 \\
Decomposition & 6 & 0.011931 & -0.156415 & -0.474735 \\
\hline \hline 50 elements & 0.018127 & -0.099119 & -0.404918 \\
\hline \hline
\end{tabular}

Table 6 Free-end displacements of helical beam; five finite elements.

\begin{tabular}{lrrrr}
\hline \hline method & $j$ & $u_{X}$ & $u_{Y}$ & $u_{Z}$ \\
\hline \hline Lagrange & 1 & 0.022301 & -0.106337 & -0.436749 \\
Scaling & 1 & 0.022912 & -0.108579 & -0.436284 \\
Decomposition & 1 & 0.014617 & -0.107658 & -0.432150 \\
\hline Lagrange & 3 & 0.020038 & -0.106751 & -0.435908 \\
Scaling & 3 & 0.020432 & -0.107457 & -0.435862 \\
Decomposition & 3 & 0.007275 & -0.114381 & -0.429273 \\
\hline Lagrange & 5 & -23.155827 & 36.496730 & 131.118915 \\
Scaling & 5 & 0.019753 & -0.107116 & -0.435719 \\
Decomposition & 5 & 0.007208 & -0.115860 & -0.429716 \\
\hline Lagrange & 6 & divergence & divergence & divergence \\
Scaling & 6 & 0.019633 & -0.107034 & -0.4356948 \\
Decomposition & 6 & 0.007311 & -0.116050 & -0.429844 \\
\hline \hline 50 elements & 0.018127 & -0.099119 & -0.404918 \\
\hline \hline
\end{tabular}

three-point elements. For such a demanding initial geometry, the benefit of scaling and wavelet functions is obvious. 
Not only that the initial geometry cannot be adequately described by Lagrangian interpolation, the numerical results diverge for higher orders of interpolation. In contrast, scaling functions and wavelets show good agreement with reference results of very fine mesh. Again we must point out the stability of scaling and wavelet based formulations with respect to order of interpolation.

\section{Conclusion}

We presented the wavelet based finite-element formulation of the consistently linearized geometrically exact spatial beam, in which the initial geometric shape of the axis and the twist of the cross-sections are arbitrary. We have established that:

(i) The scaling functions and wavelet based decomposition can be a suitable choice of shape functions in finiteelement formulations.

(ii) It is suitable to employ the explicit scaling functions and wavelets with interpolatory properties.

(iii) Scaling functions and wavelet based formulations are numerically stable for an arbitrary order of straight and curved elements. They proved to be privileged for demanding initial geometry.

(iv) The stability of the numerical solution regarding the order of interpolation indicates that such a formulation is appropriate when the mesh refinement is needed.

Acknowledgements This work was supported by the Ministry of Higher Education, Science and Technology of the Republic of Slovenia through the grant 3211-05-000549. The support is gratefully acknowledged.

\section{References}

1. J. H. Argyris, "An excursion into large rotations", Comput. Methods Appl. Mech. Eng. 32, 85-155, 1982.

2. C. K. Chui, "An introduction to wavelets", Academic Press, Boston, 1992.

3. I. Daubechies, "Ten lectures on wavelets", CBMS - NSF Regional Conference Series in Applied Mathematics, Department of Mathematics, University of Lowell, MA. SIAM: Philadelphia, PA, 1992.

4. J.G. Han, W.X. Ren and Y. Huang, "A spline wavelet finiteelement method in structural mechanics", Int. J. Numer. Methods Engng. 26, 717-730, 1988.

5. A. Ibrahimbegovic, "On finite element implementation of geometrically nonlinear Reissner's beam theory: three-dimensional curved beam elements", Comput. Methods Appl. Mech. Eng. 122, 11-26, 1995.

6. A. Ibrahimbegovic, "On the choice of finite rotation parameters", Comput. Methods Appl. Mech. Eng. 149, 49-71, 1997.

7. F. Jin, T. Q. Ye, "Instability analysis of prismatic members by wavelet-Galerkin method", Advances Engineering Software 30, 361-367, 1999.

8. A. Y. T. Leung, "Exact stiffness matrix for twisted helix beam", Finite Elements Anal. Design 9, 23-32, 1991.

9. R. H. MacNeal, R. L. Harder, "A proposed standard set of problems to test finite element accuracy", Finite Elements Anal. Design 1, 3-20, 1985.
10. J. Ma, J. Xue, S. Yang, Z. He, "A study of the construction and application of a Daubechies wavelet-based beam element", Finite Elements Anal. Design 39, 965-975, 2003.

11. J. Prestin, E. Quak, "Trigonometric interpolation and wavelet decompositions", Numerical Algorithms 9, 293-317, 1995.

12. E. Quak, "Trigonometric wavelets for Hermite interpolation", Mathematics of Computation 65, 683-722, 1996.

13. E. Reissner, "On finite deformation of space-curved beams", J. Appl. Math. Phys. 32, 734-744, 1981.

14. E. J. Sapountzakis, V. G. Mokos, "Warping Shear Stresses in Nonuniform Torsion by BEM", Comput. Mech. 30, 131-142, 2003.

15. E. J. Sapountzakis, V. G. Mokos, "A BEM Solution to Transverse Shear Loading of Beams”, Comput. Mech. 36, 384-397, 2005.

16. B. Tabarrok, M. Farshad, H. Yi, "Finite element formulation of spatially curved and twisted rods", Comput. Methods Appl. Mech. Eng. 70, 275-299, 1988.

17. G. W. Wei, D. S. Zhang, S. C. Althorpe, D. J. Kouri, D. K. Hoffman, "Wavelet-distributed approximating functional method for solving the Navier-Stokes equation", Computer Physics Communication 115, 18-24, 1998.

18. R. O. Wells, X. Zhou, "Wavelet solutions for the Dirichlet problem”, Numerische Mathematik 70, 379-396, 1995.

19. J. L. Troutman, "Variational Calculus with Elementary Convexity", Springer-Verlag, New York, 1983.

20. A. Yu, M. Fang, X. Ma, "Theoretical research on naturally curved and twisted beams under complicated loads", Comput. Struct. 80, 2529-2536, 2002.

21. Y. H. Zhou, J. Z. Wang, X. J. Zheng. "Application in beam and flat structure of wavelet Galerkin method", Applied Mathematics and Mechanics 19, 697-705, 1998.

22. D. Zupan, M. Saje, "Finite-element formulation of geometrically exact three-dimensional beam theories based on interpolation of strain measures", Comput. Methods Appl. Mech. Engrg. 192, 5209-5248, 2003.

23. D. Zupan, M. Saje, "The linearized three-dimensional beam theory of naturally curved and twisted beams: The strain vectors formulation ", Comput. Methods Appl. Mech. Engrg. 195, 4557$4578,2006$. 